[microreview]

Diamond Open Access

\title{
The metric tensor pullback
}

\author{
Open Mathematics Collaboration*†
}

May 28, 2021

\begin{abstract}
This is an article on differential geometry that connects metric tensor with its pullback [1].
\end{abstract}

keywords: metric pullback, differential geometry, quantum gravity, black hole

The most updated version of this white paper is available at https://osf.io/puhzw/download https://zenodo.org/record/4840029

\section{Introduction}

1. The motivation for this article is to try to fully understand the pullback of a metric tensor for potential applications in quantum gravity, more specifically in the black hole spacetime physics [2].

2. In the next section, we present a roadmap with the main concepts and definitions connecting metric and its pullback.

3. Some of the prerequisites needed are listed in the Supplemental Material and in [3].

*All authors with their affiliations appear at the end of this white paper.

†Corresponding author: mplobo@uft.edu.br|Open Mathematics Collaboration 


\section{Pullback metric tensor}

4. We have included here a list summarizing the mathematical definitions in order to have a global view of what is a metric tensor pullback.

5. We are considering a Riemannian manifold of two dimensions, embedded in $\mathbb{R}^{3}$.

6. In this article, the word smooth means $C^{\infty}$, or infinitely differentiable.

7. Instead of the dot product, let us replace it (due to Riemann) by an arbitrary inner product on each tangent plane of an abstract surface $M$.

8. The metric tensor $g$ is a function on all ordered pairs $(\mathbf{v}, \mathbf{w})$, where $\mathbf{v}$ and $\mathbf{w}$ are tangent vectors at the point $\mathbf{p}=\left(p_{1}, p_{2}, p_{3}\right)$ of $M \subset \mathbb{R}^{3}$.

9. In mathematical symbols, $g: \mathbb{R}^{3} \rightarrow \mathbb{R}$ given by $g_{p}(\mathbf{v}, \mathbf{w})=\langle\mathbf{v}, \mathbf{w}\rangle_{p}$, where $\langle\mathbf{v}, \mathbf{w}\rangle_{p}$ is the inner product at $\mathbf{p} \in \mathbb{R}^{3}[1]$.

10. The metric tensor is a geometric structure with a collection of inner products.

11. $g$ can also be considered a smooth, covariant, 2-tensor field [4].

12. Consider an abstract surface $A$ (see [1]) and a geometric surface $B$.

13. A geometric surface is an abstract surface composed by an inner product on each of its tangent planes [1].

14. It can be summarized as "surface + metric tensor = geometric surface" [1].

15. Let $F: A \longrightarrow B$ be a smooth map between the surfaces $A$ and $B$.

16. The map $F^{*}: \mathbb{T}^{k}(B) \longrightarrow \mathbb{T}^{k}(A)$ is the pullback by $F$, where the pullback by $\eta \in \mathbb{T}^{k}(B)$ is a smooth covariant $k$-tensor field $F^{*} \eta \in$ $\mathbb{T}^{k}(A)$. 
17. $F^{*} \eta: A \longrightarrow T^{k}(A)$, where each $p \in A$ is associated with $\left(F^{*} \eta\right)(p) \epsilon$ $T^{k}\left(T_{p} A\right)$.

18. Thus, for any vectors $v_{1}, v_{2}, \cdots, v_{r} \in T_{p} A$, we define the covariant $k$ tensor

$$
\left(F^{*} \eta\right)(p) \in T^{k}\left(T_{p} A\right)
$$

by

$$
\left(F^{*} \eta\right)(p)\left(v_{1}, v_{2}, \cdots, v_{r}\right)=\eta(F(p))\left(d F(p) v_{1}, \cdots, d F(p) v_{r}\right) .
$$

19. For $k=2$, the map $F^{*}: \mathbb{T}^{2}(B) \longrightarrow \mathbb{T}^{2}(A)$ is the pullback by $F$.

20. Let $g$ be a metric tensor on $B$, we consider $g \in \mathbb{T}^{2}(B)$.

21. Therefore, $F^{*} g \in \mathbb{T}^{k}(A)$ denotes the pullback of $g$ and it is defined by

$$
\left(F^{*} g\right)(p)\left(v_{1}, v_{2}\right)=g(F(p))\left(d F(p) v_{1}, d F(p) v_{2}\right) .
$$

22. That is,

$$
\left\langle v_{1}, v_{2}\right\rangle_{A}=\left\langle d F(p) v_{1}, d F(p) v_{2}\right\rangle_{B},
$$

for any $v_{1}, v_{2} \in T_{p} A$.

23. How can we visualize (19) graphically?

\section{Final Remarks}

24. We hope we provided all the necessary steps to understand the pullback of a metric tensor.

25. If the "hole in the black hole conjecture" [2] is correct, perhaps the pullback map $F^{*}(g)$ could provide us the fundamental means to understand the formation of a black hole. 


\section{Open Invitation}

Review, add content, and co-author this white paper [5,6].

Join the Open Mathematics Collaboration.

Send your contribution to mplobo@uft.edu.br.

\section{Open Science}

The latex file for this white paper together with other supplementary files are available in $[7,8]$.

\section{How to cite this paper?}

https://doi.org/10.31219/osf.io/puhzw

https://zenodo.org/record/4840029

\section{Acknowledgements}

+ Open Science Framework

https://osf.io

+ Zenodo

https://zenodo.org

\section{Agreement}

All authors agree with [6]. 


\section{References}

[1] O'neill, Barrett. Elementary differential geometry. Elsevier, 2006.

[2] Lobo, Matheus P. "A Hole in the Black Hole." OSF Preprints, 18 Apr. 2019. https://doi.org/10.31219/osf.io/js7rf

[3] Velleman, Daniel J. How to prove it: A structured approach. Cambridge University Press, 2006.

[4] Lee, John M. Introduction to Smooth Manifolds. New York: Springer, 2001.

[5] Lobo, Matheus P. "Microarticles." OSF Preprints, 28 Oct. 2019. https://doi.org/10.31219/osf.io/ejrct

[6] Lobo, Matheus P. "Simple Guidelines for Authors: Open Journal of Mathematics and Physics." OSF Preprints, 15 Nov. 2019.

https://doi.org/10.31219/osf .io/fk836

[7] Lobo, Matheus P. "Open Journal of Mathematics and Physics (OJMP)." OSF, 21 Apr. 2020. https://osf .io/6hzyp/files

[8] https://zenodo.org/record/4840029

[9] Leon, Steven J., Ion Bica, and Tiina Hohn. Linear algebra with applications. New York: Macmillan, 1980.

[10] Wikipedia. Metric tensor.

https://en.wikipedia.org/wiki/Metric_tensor 


\section{Supplemental Material}

\section{Definitions}

Points, dot product, norm, distance

26. $\mathbf{p}=\left(p_{1}, p_{2}, p_{3}\right)$ and $\mathbf{q}=\left(q_{1}, q_{2}, q_{3}\right)$ are points of $\mathbb{R}^{3}$.

27. The dot product of $\mathbf{p}$ and $\mathbf{q}$ is the number $\mathbf{p} \cdot \mathbf{q}=p_{1} q_{1}+p_{2} q_{2}+p_{3} q_{3}$.

28. The norm of $\mathbf{p}$ is the number $\|\mathbf{p}\|=(\mathbf{p} \cdot \mathbf{p})^{1 / 2}=\left(p_{1}^{2}+p_{2}^{2}+p_{3}^{2}\right)^{1 / 2}$.

29. $\mathbf{p}-\mathbf{q}=\left(p_{1}-q_{1}, p_{2}-q_{2}, p_{3}-q_{3}\right)$.

30. The Euclidean distance from $\mathbf{p}$ to $\mathbf{q}$ is the number $d(\mathbf{p}, \mathbf{q})=$ $\|\mathbf{p}-\mathbf{q}\|=\left(\left(p_{1}-q_{1}\right)^{2}+\left(p_{2}-q_{2}\right)^{2}+\left(p_{3}-q_{3}\right)^{2}\right)^{1 / 2}$.

\section{Relation, function}

31. Consider the sets $A$ and $B$.

32. A set $R \subseteq A \times B$ is a relation from $A$ to $B$.

33. $A \times B=\{(a, b) \mid a \in A \wedge b \in B\}$ is the Cartesian product.

34. A relation $f \subseteq A \times B$ is a function from $A$ to $B$, written $f: A \rightarrow B$, if $\forall a \in A \exists ! b \in B((a, b) \in f)$.

35. $f$ is one-to-one if $\neg \exists a_{1} \in A \exists a_{2} \in A\left(f\left(a_{1}\right)=f\left(a_{2}\right) \wedge a_{1} \neq a_{2}\right)$.

36. $f$ is onto if $\forall b \in B \exists a \in A(f(a)=b)$.

37. $f$ is a bijection if it is one-to-one and onto.

38. if $f: A \rightarrow B$ is bijective, then $f^{-1}: B \rightarrow A$ is the inverse of $f$. 


\section{Vector space, inner product}

39. $\mathbb{R}^{3}$ is a vector space over the real numbers for it satisfies the axioms for a vector space [9].

40. An inner product is a function that assigns two vectors to a number.

41. The number $\langle\mathbf{v}, \mathbf{w}\rangle$ is an inner product between the pair of vectors $\mathbf{v}, \mathbf{w}$ in the vector space $V$.

Open subset, neighborhood, one-to-one mapping

42. $D \subseteq \mathbb{R}^{2}$ is an open subset of $\mathbb{R}^{2}$ iff $\forall d \in D, \exists c>0, c \in \mathbb{R}$ such that the interval $(d-c, d+c) \subseteq D$.

43. $c$ is called a neighborhood of $d$.

44. $\mathbf{x}: D \rightarrow \mathbb{R}^{3}$ is a one-to-one mapping (function) called a coordinate patch.

45. A proper patch is a coordinate patch that has the inverse function $\mathbf{x}^{-1}$.

\section{Surface, differentiability}

46. A surface in $\mathbb{R}^{3}$ is a subset of $\mathbb{R}^{3}$ that is smooth and two-dimensional, i.e., that satisfies (44) and (45).

47. Let a surface be a set $M$, a collection of points.

48. Consider a one-to-one function $\mathbf{x}: D \rightarrow M$.

49. (48) is an abstract patch in $M$.

50. Let $\mathcal{P}$ be a collection of abstract patches. 
51. The covering axiom states that the images of the patches in $\mathcal{P}$ cover $M$.

52. The smooth overlap axiom states that $\forall \mathbf{x}, \mathbf{y} \in \mathcal{P}, \mathbf{y}^{-1} \mathbf{x}$ and $\mathbf{x}^{-1} \mathbf{y}$ are Euclidean differentiable and defined on open sets of $\mathbb{R}^{2}$.

53. Euclidean differentiable means differentiable on Euclidean space.

\section{Ordered triples, Euclidean space}

54. The set of all ordered triples of real numbers $\mathbf{p}=\left(p_{1}, p_{2}, p_{3}\right)$, called points, is a Euclidean 3 -space $\mathbb{R}^{3}$.

55. Let $f$ be a real-valued function on $\mathbb{R}^{3}$.

56. Real-valued function means that its codomain is a cartesian product of $\mathbb{R}$.

57. $f$ is differentiable if all partial derivatives of $f$, of all orders, exist and are continuous.

58. $f$ is said to be infinitely differentiable, or smooth, or of class $C^{\infty}$.

\section{Metric tensor}

59. "A metric tensor is a type of function which takes as input a pair of tangent vectors $v$ and $w$ at a point of a surface (or higher dimensional differentiable manifold) and produces a real number scalar $g(v, w)$ in a way that generalizes many of the familiar properties of the dot product of vectors in Euclidean space." [10]

\section{Tensors and Tensor Fields}

60. Consider a finite-dimensional real vector space $E$. 
61. A covariant $k$-tensor on $E$ is a real-valued multilinear function of $k$ elements of $E$

$$
T: \underbrace{E \times \cdots \times E}_{k \text { times }} \longrightarrow \mathbb{R} .
$$

62. $T^{k}(E)$ denotes the vector space of all covariant $k$-tensors on $E$.

63. Let $A$ be a smooth abstract surface.

64. We define the bundle of covariant $k$-tensors on $A$ by

$$
T^{k} A=\prod_{p \in A} T^{k}\left(T_{p} A\right),
$$

this bundle is called tensor bundle on $A$.

65. A smooth section of a tensor bundle is called a smooth tensor field on $A[4]$.

66. $\mathbb{T}^{k}(A)=\left\{\right.$ smooth sections of $\left.T^{k} A\right\}$ denotes the real vector space of smooth sections of the bundle $T^{k} A$.

67. If $\eta \in \mathbb{T}^{k}(A)$, then $\eta$ is a map

$$
\eta: A \longrightarrow T^{k} A,
$$

called smooth covariant $k$-tensor field on $A$, where each $p \in A$ is associated with the covariant $k$-tensor $\eta(p) \in T^{k}\left(T_{p} A\right)$.

\section{The Open Mathematics Collaboration}

Matheus Pereira Lobo (lead author, mplobo@uft.edu.br) ${ }^{1}$

https://orcid.org/0000-0003-4554-1372

Alvaro Julio Yucra Hancco ${ }^{1}$

João Pedro Almeida Sales ${ }^{1,2}$

${ }^{1}$ Federal University of Tocantins (Brazil)

${ }^{2}$ Colégio da Polícia Militar 